\title{
Research and Application of "Internet+" Intelligent Heating System for Central Heating
}

\author{
Pingping Huang*, Xinrong Yan, Kaixuan Guo \\ Huadian Electric Power Research Institute Co, Ltd, Hangzhou, China \\ Email address: \\ 1542231909@qq.com (Pingping Huang), xinrong-yan@chder.com (Xinrong Yan), kaixuan-guo@chder.com (Kaixuan Guo) \\ ${ }^{*}$ Corresponding author
}

\section{To cite this article:}

Pingping Huang, Xinrong Yan, Kaixuan Guo. Research and Application of “Internet+" Intelligent Heating System for Central Heating. American Journal of Engineering and Technology Management. Vol. 6, No. 5, 2021, pp. 76-81. doi: 10.11648/j.ajetm.20210605.11

Received: August 23, 2021; Accepted: September 9, 2021; Published: September 15, 2021

\begin{abstract}
At present, China's central heating industry is developing rapidly. It is an important direction for the transformation and upgrading of the energy industry to use the advantages of advanced information and communication technology and Internet platform to promote the intelligent energy production, promote the interconnection of various types of energy flow network and the coordinated transformation of various energy forms. In the framework of the era of industrial Internet of Things, the introduction of "Internet + " technology helps to improve the production, operation, management and service level of central heating system from the system engineering level. The "Internet + " intelligent heating system in the field of central heating integrates heating physical system and information system deeply, forming a new application mode of "digital twin". It takes "heat source -- heat network -heat station -- user heat transfer" as the main line and adopts B/S architecture. It consists of data perception layer, data acquisition layer, data access layer, business logic layer, presentation layer and terminal access layer. In a "Internet + " intelligent heating platform demonstration construction applications, through the construction of entire network hydraulic balance analysis, the source network integration of collaborative scheduling, energy consumption statistics and analysis, management fees management, customer service management subsystem module, such as the heating index to reduce energy consumption, operating cost savings, improve work efficiency and service quality improvement has made significant achievements.
\end{abstract}

Keywords: “Internet+", Intelligent Heating System, Source-Network Integration, B/S Framework, Digital Twin

\section{Introduction}

At present, Chinese central heating industry rapid development. In the production and operation of thermal enterprises, there are problems such as the overall low level of intelligence and extensive management mode: first, a considerable part of central heating systems have not yet realized the remote transmission and remote control of important parameters, lack of data support for online diagnosis and real-time analysis, hydraulic imbalance and cold and heat imbalance often occur; Second, due to the lack of guarantee for data collection and data sharing, it is difficult to evaluate the index economy and operation timeliness before and after the implementation of intelligent heat network. Third, the charging customer service system function is not perfect, hot user service can't meet the requirements of the Internet information age; Fourth, in the established heating operation management platform, there are information barriers between heat source, heat network, heat station and users, and the operation adjustment strategy is mostly based on local system consideration, which fails to realize the cooperative control of source and network [1].

In the continuous expansion of the application scale of cogeneration units, it is necessary to build intelligent control system under the Industrial Internet of Things. The extension of the Internet of things is based on the Internet and extension, using advanced information and communication technology and the advantage of the Internet platform, promote the intelligent energy production, promote a variety of types can flow the network connectivity and a variety of energy form cooperative transformation, construction of "low-carbon clean, safe and efficient" modern energy system, is the important direction of the transformation and upgrading of the energy industry. In the context of the rapid development of advanced industrial Internet of Things information technology and the concept of "consumption, supply, technology and system" of energy on the 
agenda, China's central heating enterprises need to make use of "Internet + " technology to form an industrial Internet of Things system in a modern, digital and intelligent way. To improve the production, operation, management and service level of central heating system at the system engineering level.

Intelligent heating is still in the process of rapid development, the concept of continuous evolution, industry researchers from different perspectives and different levels of research and exploration. From the initial information upgrading of traditional heating management business, to remote monitoring and transformation of the control system of the heating station, to the independent application of each business management system, and now to explore and combine advanced information technology to build an integrated platform of heating operation, the connotation of intelligent heating has gradually become clear.

This paper focuses on the research and application of "Internet + " intelligent heating system in central heating. First of all, the central heating industry "Internet + " demand analysis, the research and development of "Internet +" intelligent heating system is important; Then, elaborate the "Internet + " intelligent heating system construction content; Finally, an application example of "Internet + " intelligent heating platform is presented.

\section{2. "Internet +" Intelligent Heating System}

Industrial iot is an important part of a new generation of information technology, is also important stage of development of information age, namely based on the extension and expansion of the Internet to realize the information exchange between different items, namely " Internet of everything", and "Internet + " intelligent heating system is industrial iot in a typical application in the field of central heating.

"Internet + " intelligent heating system refers to a new intelligent heating network based on heating automation and information through the deep integration of physical system and information system. The mechanism model is combined with the data-driven model to realize the "digital twin", which is the application kernel of industrial Internet of Things system. An information processing system that integrates advanced technologies such as data identification, data mining and artificial intelligence. The significance of the "Internet + " intelligent heating system is that it can provide whole-process intelligent decision support for the operation and management of the heating system [2, 12-15].

\section{3."Internet +" Intelligent Heating System Construction Content}

"Internet +" intelligent heating system with "heat source heat supply network - transfer station - the user thermal transfer" as the main line, covering the civil heating and industrial steam supply two kinds of business model, with the lean operation management service as the core, the unified deployment of network resource database, through running, collect fees, customer service, energy consumption, management and other business applications of data channels, Realize the full interactive call of the data of each functional subsystem in the platform, and promote the online real-time collaboration of various services. "Internet + " intelligent heating system will truly realize the demand scenario, scene online, online business, multi-business integration.

The platform adopts $\mathrm{B} / \mathrm{S}$ architecture, including data awareness layer, data acquisition layer, data access layer, business logic layer, presentation layer and terminal access layer [3-7]. The data sensing layer includes the automation equipment of the Internet of Things needed for the construction of the intelligent heating system, which is used to complete the operation instructions and task commands in the intelligent heating platform. Data acquisition layer and data access layer mainly through the Internet platform to achieve the whole process of central heating system data acquisition, intelligent regulation and control functions; The business logic layer is the management and decision center of the intelligent heating platform, providing information system support and auxiliary decision support for the production management of thermal enterprises, and providing good customer service and "Internet + " experience for thermal users. The presentation layer and terminal access layer are the display window of the platform and provide good human-computer interaction function for the operators. The application architecture of "Internet + " intelligent heating system in thermal enterprises is shown in Figure 1.

\subsection{Data Awareness Layer}

Heat source side: electric control valve is installed in the first station to coordinate with the circulating pump frequency converter to adjust the heat transfer in the first station, and measuring devices such as temperature, pressure and flow are installed at each output pass to calculate the heat.

Heat station side: add and improve relevant parameter measurement points (temperature, pressure, flow, liquid level, heat, etc.), safety equipment (water running alarm, camera, intercom device), control device (solenoid valve, regulating valve, frequency converter, etc.), measuring device (water meter, electricity meter, heat meter) in the station.

Thermal user side: install indoor temperature measurement device in the designated residential area; For the residential areas charging according to the measurement, add the on-off valve and heat meter into the household to realize heating on demand.

\subsection{Data Acquisition Layer}

The relevant parameters of the data sensing layer equipment are transmitted to the heat network monitoring system through THE Ethernet communication technology such as GPRS/ 5.8g /MSTP, so as to realize the real-time data acquisition and remote centralized regulation of all heat stations. The parameters of the temperature measuring device, household heat meter and on-off valve in the cell are 
transmitted to the PLC receiving module of the heating station by M-BUS mode or NB-LOT wireless communication mode, and then to the monitoring center of the heat network by the Ethernet special line [8].

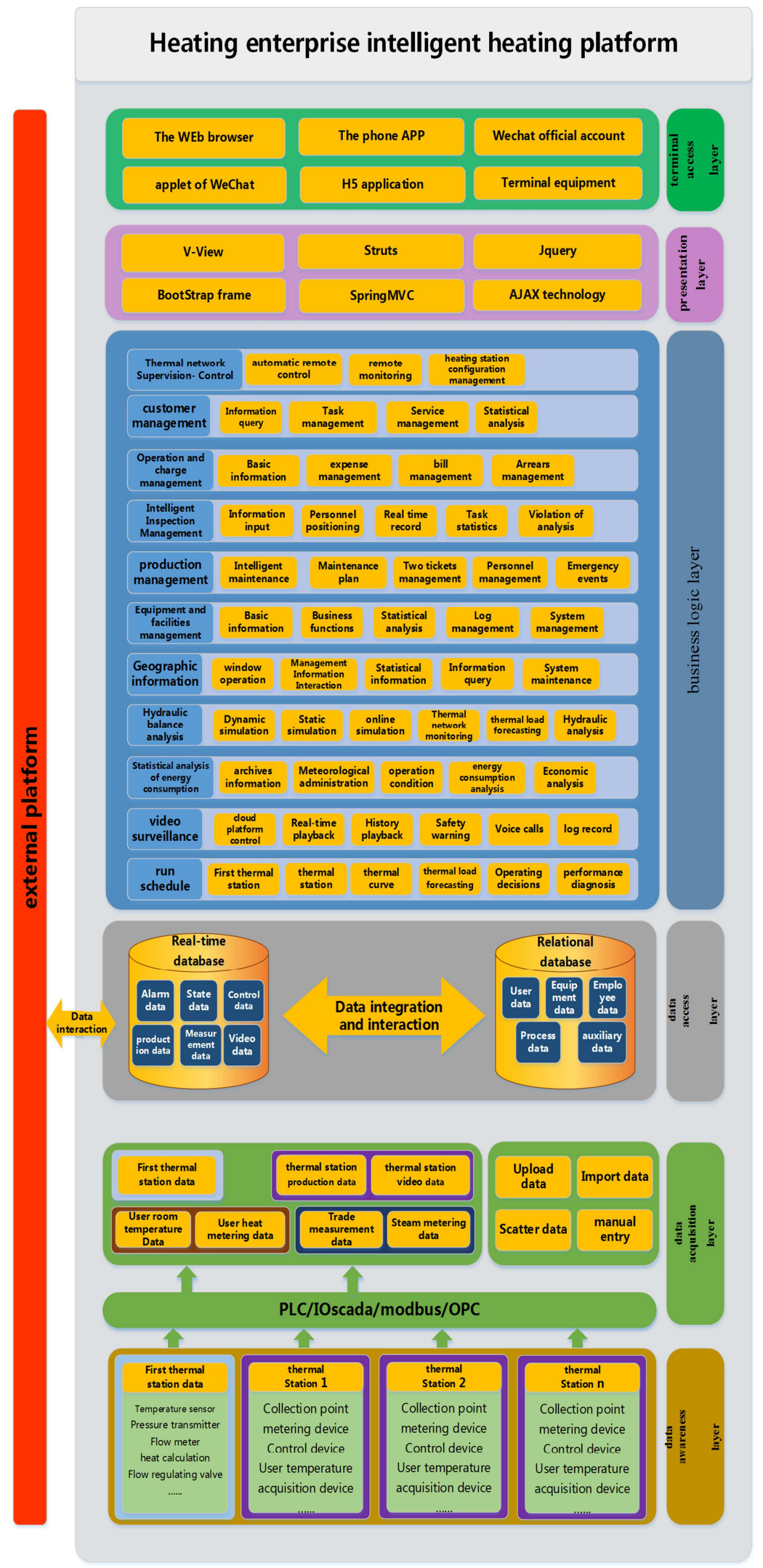

Figure 1. Application architecture of "Internet +" intelligent heating system for thermal enterprises. 


\subsection{Data Access Layer}

The data access layer contains the real-time database and the relational database, which receives and stores all the data from the data acquisition layer.

The real-time database is used to store the real-time data in the process of production and operation, and the source is mainly from the functional modules of data monitoring, gate measurement, trade settlement and so on of the first station/heat station/heat user. Different modules have different requirements for collection accuracy, which can be divided into "millisecond level" and "minute level".

Relational database is used to store the filling, configuration parameters and other relational data, as a real-time data platform to improve and supplement.

\subsection{Business Logic Layer}

The business logic layer is the core content of the platform, which is used for logical judgment and execution operations in the platform business work. After receiving the user instructions of the presentation layer, the data access layer is connected to realize the instruction transmission among the three layers, carry out logical processing of the received data, complete the modification, acquisition, deletion and other functions of the data, and finally feedback the processing results to the presentation layer.

The business logic layer mainly includes Analysis of hydraulic balance subsystem, Operation scheduling subsystem, Thermal network Supervision- Control subsystem, Statistical analysis of energy consumption subsystem, Operation and charge management subsystem, Customer service management subsystem, Production management subsystem, Equipment and facilities management subsystem, Intelligent Inspection Management subsystem, Geographic information subsystem.

\subsection{The Presentation Layer and Terminal Access Layer}

The presentation layer and terminal access layer provide a unified business application operation interface and information display window for the "Internet + " intelligent heating platform to realize the input and output of platform data, and the communication and display of business data among subsystem modules.

The presentation layer adopts HTML page or JSP page, data interaction and terminal cache by means of AJAX asynchronous request, and user friendly operation experience and smooth data display are guaranteed by technologies such as custom components. Supported by the sound logic computing technology of the background business logic layer, The presentation layer can support the customization and change of various external pages and forms, providing high-quality operation interface and information display window for intelligent heating platform.

The terminal access layer is the appearance layer of the platform and is responsible for providing users with a friendly human-computer interface. $\mathrm{B} / \mathrm{S}$ architecture for different types of users to access. Internal users can access the platform through WEB interface and mobile management client. Hot users access the platform front application server through mobile phone applications or wechat public platform and query the heating business data related to hot users themselves.

\section{Application Example of "Internet +" Intelligent Heating Platform}

Through the demonstration construction and application of the "Internet + " intelligent heating platform in a grassroots thermal power enterprise integrating the source and network, remarkable results have been achieved in reducing energy consumption of heating index, saving operating cost, improving work efficiency and improving service quality.

At the level of data perception, by installing the Internet of Things regulating valve, water pump inverter and automatic control system in the thermal station, the monitoring and measurement points of the main operating parameters in the station are improved, video monitoring equipment is added, and all operating parameters collected in the thermal station are transmitted to the intelligent heating platform in a unified manner by using the leased Ethernet network of the operator. The dispatching center can realize the monitoring and remote control of the running state of the equipment in the thermal station, so as to realize the unattended status of the thermal station.

On the platform business analysis level, the whole network hydraulic balance analysis subsystem, source network integration collaborative scheduling subsystem, energy consumption statistics and analysis subsystem, operation charge management subsystem, customer service management subsystem modules are built.

\subsection{The Whole Network Hydraulic Balance Analysis Subsystem}

According to the actual topological structure of the pipe network, the thermal network model was established through the mechanism analysis of the thermal system and the modeling method driven by historical big data, so as to realize the functions of offline simulation calculation and online operation condition optimization. By comparing the soft sensor results calculated by the model with the actual operation parameters of the heat network, the operation scheduling is guided and the control strategy is optimized.

\subsection{Source Network Integration Collaborative Scheduling Subsystem}

Historical operation data, outdoor meteorological parameters according to the network, network inertia, building features, the end of the heating form, users multi-dimensional factors such as room temperature forecast heat load, the real-time calculate the thermal station current best secondary heating water temperature, make each unique 
adjustment curve heating station, implement of "one stop one policy " precision control. To user side indoor temperature for the final control objectives, the heat exchange station secondary water supply temperature, so the heat exchange station a network side electric regulating valve opening, heat supply network first a traceability related parameters, such as water temperature, extraction cogeneration unit and a network of water supply temperature to coordinate the relationship of change, thus forming the source thermal regulation model of network integration. The control strategy of "one station one policy" of the source network cooperative scheduling subsystem is shown in Figure 2.

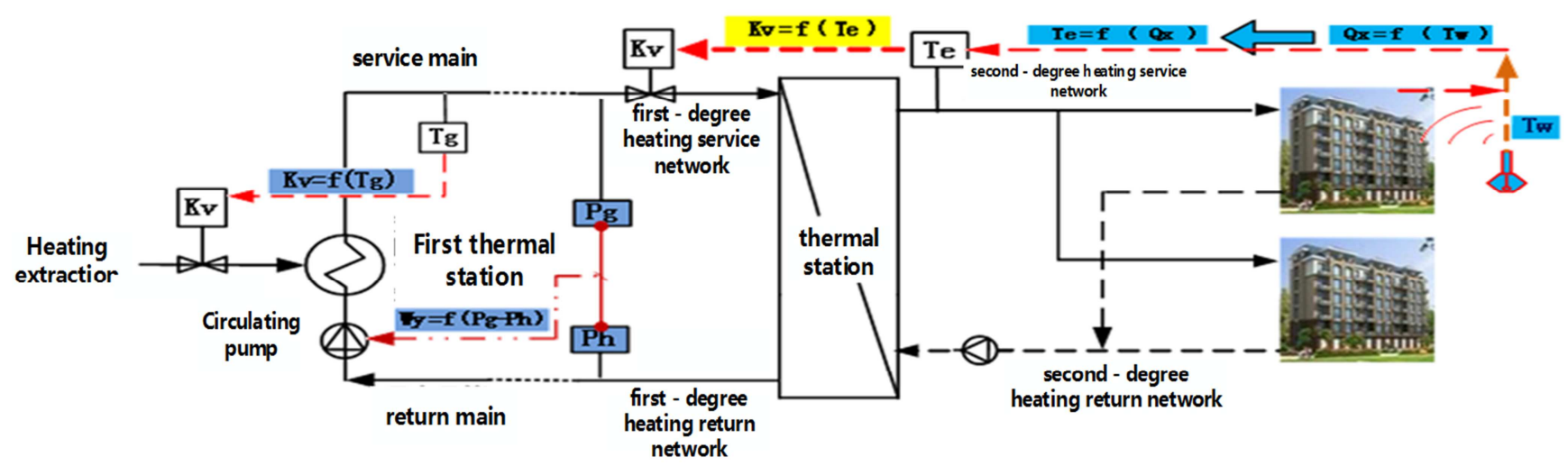

Figure 2. "One station one policy" control strategy of source network cooperative scheduling subsystem.

Note:

(1) Tw: Monitor the indoor temperature

(2) $T_{e}$ : Water service temperature of second-degree network of thermal station

(3) $K_{v}$ : Electric regulating valve opening of thermal station

(4) $P_{g}-P_{h}$ : Most adverse loop pressure differential

(5) $W_{y}$ : Circulating pump frequency of the first thermal station

(6) $T_{g}$ : Water temperature of service main

(7) $K_{v}$ : Opening of heating extraction steam regulating valve of cogeneration unit

\subsection{Statistical Analysis of Energy Consumption Subsystem}

Through the establishment of heating energy consumption index calculation model, using the operation of the heating system of various types of data, water, electricity, heat and other energy consumption index calculation, and in various forms such as graphical statements, in different dimensions to analyze and present. Further, combined with price factors such as water price, electricity price and heat price, refine the marginal contribution of heating, increase the cost information of labor and materials involved in production, establish the influence relationship between energy saving effect and economic benefit, and promote the optimization and transformation of energy saving of heating system from the perspective of economic benefit priority.

\subsection{Operation and Charge Management Subsystem}

Through the establishment of one-stop heating charging solution, supplemented by Banks, WeChat, etc, in pay treasure payment methods, and ensure the interface, electronic invoice system, etc., associated customer service subsystem, realize across different departments, the system linkage charge management, mainly including and inspection management, production scheduling, the linkage of the customer service management coordination [9]. Solve problems such as confusion of user data, unclear accounts, low rate of collection, untraceable historical information and so on, improve the efficiency of operation and charge and customer satisfaction.

\subsection{Customer Service Management Subsystem}

Integrated service hall, telephone, network, as well as social supervision, competent authorities and other diversified customer service request channels, smooth information transmission mechanism. According to the type and urgency of the work order, the customer service center will send the work order and visit the customer. The mobile terminal is used to complete personnel positioning, remote order receiving, remote supervision, work order processing feedback, and establish a closed-loop management mechanism consisting of order sending, processing, supervision, feedback, return visit and other links, so as to achieve rapid response to customer needs. Collect all kinds of service information of the customer service management system in the whole process, including key indicators such as customer service request response rate, customer service request completion rate, seat utilization rate and hotline connection rate, and establish a comprehensive evaluation mechanism $[10,11]$.

\section{Conclusion and Recommendations}

With the rapid development of energy Internet technology, the integration of advanced Internet information technology and central heating system is becoming deeper and more widely used. In the "Internet $+"$ technology, under the blessing of the meet heating enterprise data remote transmission, remote control operation, on the basis of the 
future can be further "to do real bottom, activate the cloud", to further promote grass-roots heating enterprise saving energy and reducing consumption, mass transfer efficiency, to achieve thermal "production - losing - with -" sale process refinement, standardization, intelligent management, steadily improving customer service levels, In order to meet the needs of customers under the "Internet + " environment, improve the value of enterprise heating brand.

\section{References}

[1] HUADIAN ELECTRIC POWER RESEARCH INSTITUTE. (2020). Typical Cases of Energy-saving Retrofits for Combined Heat and Power. CHINA ELECTRIC POWER PRESS, 16-21.

[2] CHINA DISTRICT HEATING ASSOCIATION. (2019). Blue Book of Heating in China 2019 Smart Urban Heating. China Architecture and Building Press, 60-176.

[3] Jian Jiang, Fei Wang, Xiangguang Han. (2020). Based on artificial intelligence to achieve the heating system "source website household" linkage control. District heating. 2020 (5): 125-131.

[4] Xiumu Fang., Dayi Yang., Zhigang Zhou, (2019). Connotation and Goal of Smart Heating. GAS \& HEAT, 39 (7), 1-7.

[5] China Electric Power Research Institute. (2013). Application of cloud computing and Internet of Things technology in power system. China Electric Power Press.

[6] Chunlei Chai (2020). Research and architecture design of intelligent heating system. Internet of Things Technology; 2020 (5): 109-111.
[7] Yuqiang Cao, Jinan Shen. (2002). Energy saving intelligent heating control system. Industrial Instrumentation \& Automation. 2002 (1): 44-46.

[8] Zhimin Wang, Songran Li, (2020). The practical exploration and optimization of intelligent heating customer service, District heating, 115-117.

[9] Rui Yang. (2020). Construction and implementation of intelligent, digital and network heating system. Huadian Technology, 11, 97-99.

[10] Wei Zhong, Lijun Zheng, Zitao Yu, Xiaojie Lin. (2020). Smart heat-supply roadmap based on digital twin. Huadian Technology, 2020 (11), 1-5.

[11] Chen Shen, Mengshuo Jia, Yin Chen, et al. (2020). Digital twin og the energy internet and its application, Journal of Global Energy Interconnection, 2020, 3 (1), 1-13.

[12] WEI Z, CHEN J Y, ZHOU Y, et al,. (2019). Network flexibility study of urban centralized heating system: Concept, modeling and evaluation, Energy, 2019, 177, 334 - 346.

[13] Zhongkai Yi, Zhimin Li,. (2019). Combined heat and power dispatching strategy considering heat storage characteristics of heating network and thermal inertia in heating area. Power System Thchnology, 2018, 42 (5), 1378-1384.

[14] Fei Tao, Qinglin Qi., Lihui Wang, et al. (2019). Digital twins and cyber-physical systems toward smart manufacturing and Industry 4.0: Correlation and comparison. Engineering, 2019, 5 (4): 132-149.

[15] Shenglan Jing., Fei Wang, Yonggang Lei. (2015). Review on heating load prediction method. Building Energy \& Environment, 2015, 34 (4): 31-35. 